\title{
Detection of macrolide resistance genes in culture-negative specimens from Bangladeshi children with invasive pneumococcal diseases
}

Md. Hasanuzzaman ${ }^{a}$, Roly Malaker ${ }^{\text {a }}$, Maksuda Islam ${ }^{\text {a }}$, Abdullah H. Baqui ${ }^{\text {}}$, Gary L. Darmstadt ${ }^{c}$, Cynthia G. Whitney ${ }^{d}$, Samir K. Saha ${ }^{a, e, *}$

${ }^{a}$ Child Health Research Foundation, Department of Microbiology, Dhaka Shishu Hospital, Dhaka 1207, Bangladesh

${ }^{\mathrm{b}}$ International Health, Johns Hopkins Bloomberg School of Public Health, Baltimore, $M D, U S A$

${ }^{\circ}$ Department of Pediatrics, Stanford University School of Medicine, Stanford, CA, USA

${ }^{d}$ Respiratory Diseases Branch, US Centers for Disease Control and Prevention, Atlanta, GA, USA

e Bangladesh Institute of Child Health, Dhaka Shishu Hospital, Dhaka, Bangladesh

ARTICLE INFO

Article history:

Received 7 June 2016

Accepted 11 November 2016

Keywords:

Macrolide resistance gene 
Invasive pneumococcal disease

Culture-negative

Bangladesh

* Corresponding author. Tel.: +880 171346 1254; fax: +880 29128308.

E-mail address: samirk.sks@gmail.com (S.K. Saha). 


\section{ABSTRACT}

Objectives: In recent years, an increasing prevalence of macrolide resistance among pneumococci in Bangladesh has been observed. However, the scenario remains incomplete, as few isolates $(<1 \%)$ are available from pneumonia cases and most pneumococcal meningitis cases $(>80 \%)$ are culture-negative. This study optimised a triplex PCR method to detect macrolide resistance genes (MRGs) (mefA and ermB) and cpsA from culture-negative pneumococcal cases to predict the prevalence and level of macrolide resistance.

Methods: The presence of MRGs among pneumococcal strains $(n=153)$ with a wide range of erythromycin MICs ( $<0.5$ to $\geq 256 \mathrm{mg} / \mathrm{L}$ ) was determined by PCR. Triplex PCR was validated by simultaneous detection of MRG(s) and $\operatorname{cps} A$ in culture-negative clinical specimens and corresponding isolates. The known impact of the presence of specific MRG(s) on MICs of strains was used to predict the MICs of non-culturable strains based on the presence/absence of MRG(s) in the specimens.

Results: None of the erythromycin-susceptible isolates possessed any of the MRGs, and all non-susceptible strains had $\geq 1 \mathrm{MRG}$. MICs were $2-16 \mathrm{mg} / \mathrm{L}$ and $\geq 256 \mathrm{mg} / \mathrm{L}$ for $93 \%$ of strains with mefA and ermB, respectively, whereas $100 \%$ of isolates with both genes had MICs $\geq 256 \mathrm{mg} / \mathrm{L}$. PCR for body fluids showed 100\% concordance with corresponding isolates when tested for $\mathrm{MRG}(\mathrm{s})$ in parallel.

Conclusions: Erythromycin MICs can be predicted for non-culturable strains with 93$100 \%$ precision based on detection of $e r m B$ and/or mefA. This method will be useful for establishing comprehensive surveillance for macrolide resistance among pneumococci, specifically in the population with prior antibiotic use. 


\section{Introduction}

Streptococcus pneumoniae (pneumococcus) is a major bacterial pathogen responsible for ca. 476000 deaths in children aged 0-59 months, $>90 \%$ of which occur in developing countries [1]. Despite the remarkable success of pneumococcal conjugate vaccines (PCVs), appropriate treatment of pneumococcal disease remains salient as no vaccine is $100 \%$ effective and the available vaccines fail to cover all infection-causing serotypes [2]. In Bangladesh, $<50 \%$ of pneumococcal meningitis cases are caused by vaccine serotypes [3,4]. Clinical management of pneumococcal disease is complicated due to the worldwide emergence of resistance in $S$. pneumoniae to commonly used antibiotics, specifically to trimethoprim/sulfamethoxazole, penicillin and, recently, macrolides [5,6]. In Bangladesh, we reported macrolide resistance among $1 \%$ of pneumococci in 2008 and an increased rate of 46\% in 2014 [7].

Similar to findings by other groups, macrolide resistance of our pneumococcal isolates is caused by acquisition of ribosomal RNA methylation mediated by the ermB gene and/or efflux pump-mediated resistance due to mefA [8,9]. However, this finding is based on limited information, as the rate of isolation of pneumococcus from pneumonia cases is low $(<1 \%)[10,11]$ and thus surveillance of the invasive pneumococcal disease burden, serotype distribution and antibiotic resistance relies mostly on meningitis isolates [12]. However, cerebrospinal fluid (CSF) specimens from children with meningitis are often sterilised by antibiotics received before cultures are collected; only $22 \%$ of pneumococcal meningitis cases are detected by culture [3]. Although Binax or PCR could detect culture-negative cases as pneumococcal, the antibiogram for the 
infecting pneumococci remains unknown, leading to fragmented knowledge about true rates of resistance.

To overcome this limitation, we aimed to optimise PCR-based detection of macrolide resistance genes (MRGs) from culture-negative invasive pneumococcal cases in body fluids and to use the minimum inhibitory concentrations (MICs) of known isolates with corresponding resistance genes to predict the prevalence and level of resistance among the culture-negative (Binax/PCR-positive) pneumococcal cases.

\section{Materials and methods}

For this study, 153 S. pneumoniae isolates were retrospectively selected, which were prospectively collected from patients with different clinical manifestations. The cases were from three World Health Organization (WHO) Invasive Bacterial Vaccine Preventable Diseases (IB-VPD) sentinel sites and a rural population-based site (see Supplementary Table S1). All of the isolates were further confirmed at a WHO IB-VPD reference laboratory (Dhaka Shishu Hospital, Dhaka, Bangladesh). MICs were determined by Etest and were interpreted according to Clinical and Laboratory Standards Institute (CLSI) guidelines [13]. Isolates included in the study had varied levels of susceptibility to erythromycin; 121 were erythromycin-non-susceptible (MIC range, $2.0 \mathrm{mg} / \mathrm{L}$ to $\geq 256 \mathrm{mg} / \mathrm{L}$ ) and 32 were erythromycin-susceptible ( $\mathrm{MIC}<0.5 \mathrm{mg} / \mathrm{L}$ ). These strains were tested by duplex PCR to detect the presence of ermB and mefA genes following standard methods [8]. 
The same PCR method was used, with minor modifications, for direct detection of MRGs in clinical specimens. In brief, unlike DNA extraction from isolates by boiling, a QIAamp ${ }^{\circledR}$ DNA Mini Kit (QIAGEN, Hilden, Germany) was used for extraction of DNA from CSF and ascitic fluid according to the manufacturer's instructions. PCR amplicons were produced by an initial cycle at $95^{\circ} \mathrm{C}$ for $15 \mathrm{~min}$, followed by 35 cycles of denaturation at $94{ }^{\circ} \mathrm{C}$ for $1 \mathrm{~min}$, annealing at $58^{\circ} \mathrm{C}$ for $1 \mathrm{~min}$ and extension at $72{ }^{\circ} \mathrm{C}$ for 1 min, with a final extension at $72{ }^{\circ} \mathrm{C}$ for $10 \mathrm{~min}$. PCR products were electrophoresed in a $1.5 \%$ agarose gel at $100 \mathrm{~V}$ for $50 \mathrm{~min}$, were stained with SYBR ${ }^{\circledR}$ Safe (Invitrogen, Carlsbad, CA) and were visualised using an ultraviolet transilluminator (Gel Doc XR+ System; Bio-Rad, Hercules, CA).

For concurrent confirmation of the presence of pneumococcus, an additional primer set for the capsular gene (cpsA) of pneumococcus was added [14]. This triplex PCR (mefA, ermB and $\operatorname{cps} A$ ) was first applied on the specimens (CSF and ascitic fluid) of cultureconfirmed cases. After confirming the reproducibility of the method with known isolates and the corresponding specimens, the triplex PCR was then applied on previously characterised culture-negative specimens (CSF or ascetic fluid) from invasive pneumococcal (antigen- and PCR-positive) cases for simultaneous identification of the pneumococcal $\operatorname{cps} A$ gene and MRGs.

The pneumococcal DNA load from these culture-negative cases were further determined by quantitative PCR (qPCR) assay for the lytA gene according to a standard protocol [15]. 
The MICs of isolates were associated with the resistance gene(s) present and this association was used to predict the possible MICs (ranges) among the culture-negative cases. Precision of the predicted MIC (or range) of a non-culturable case was interpreted by assigning a positive predictive value (PPV) as calculated using the formula PPV $=(A / A+B) \times 100$, where $A$ is the number of positive observations and $B$ is the number of negative observations by any particular test.

CSF specimens were tested for the presence of antibiotic activity following procedures described previously [16]. In addition to the test for the presence of antibiotic activity, the study team questioned caregivers on the duration of antibiotic therapy prior to collection of specimens at the hospital.

\section{Results}

All erythromycin-non-susceptible isolates $(n=121)$ possessed either ermB, mefA or both $(58 \%, 36 \%$ and $6 \%$, respectively). In contrast, none of the susceptible isolates ( $n=$ 32) possessed either gene (Fig. 1a; Table 1). The ermB gene was most prevalent in isolates with a high level of resistance; MICs for ermB-positive strains ranged from 64 $\mathrm{mg} / \mathrm{L}$ to $\geq 256 \mathrm{mg} / \mathrm{L}$, and $>93 \%(65 / 70)$ had an MIC $\geq 256 \mathrm{mg} / \mathrm{L}$. The mefA gene was associated with a low level of resistance; MICs ranged from $2 \mathrm{mg} / \mathrm{L}$ to $24 \mathrm{mg} / \mathrm{L}$, and 93\% (41/44) had an MIC $\leq 16 \mathrm{mg} / \mathrm{L}$ (Table 1). Isolates carrying dual resistance genes $(n$ = 7) invariably had an MIC $\geq 256 \mathrm{mg} / \mathrm{L}$. Direct triplex PCR of corresponding CSF specimens from culture-positive pneumococcal cases $(n=10)$ generated identical 
results to their isolates (Fig. 1b) with respect to the presence of MRGs (ermB and mefA) and $\operatorname{cps} A$.

Subsequently, 68 specimens (61 CSF and 7 ascitic fluid) from culture-negative (antigenpositive) invasive pneumococcal cases with unknown susceptibility results were subjected to triplex PCR to identify MRGs. All cases except three were positive for cpsA. The cpsA-negative, antigen-positive cases were serotype 38, as detected by multiplex PCR for serotyping [17]. Nineteen cases (28\%) had only the $\operatorname{cps} A$ gene with no detection of MRGs. The remaining 49 cases $(72 \%)$ had either or both of the MRGs detected (Fig. 2); 38\% (26/68) were positive for mefA, 22\% (15/68) for ermB and 12\% $(8 / 68)$ for both ermB and mefA. All of these cases were positive for the lytA gene with a CT value of $<31$ (mean 24.7 and median 25.4), and there was no significant difference $(P=0.3923)$ in lytA CT value among MRG-positive (mean 24.9 and median 25.6) and MRG-negative cases (mean 24.1 and median 24.7).

In addition to labelling the non-culturable specimens as having susceptible or nonsusceptible pneumococci, detection of MRGs can also facilitate the classification of these cases into the following groups based on the association of the MRGs among the isolates (Table 1):

1. no MRGs: susceptible with $\mathrm{MIC}<0.5 \mathrm{mg} / \mathrm{L}(\mathrm{PPV}=100 \%)$;

2. presence of mefA gene: non-susceptible with MIC range of $2-24 \mathrm{mg} / \mathrm{L}$; most likely MIC within range $2-16 \mathrm{mg} / \mathrm{L}(\mathrm{PPV}=93 \%)$; 
3. presence of $\mathrm{ermB}$ gene: non-susceptible with MIC range of $64 \mathrm{mg} / \mathrm{L}$ to $\geq 256$ $\mathrm{mg} / \mathrm{L} ;$ most likely MIC $\geq 256 \mathrm{mg} / \mathrm{L}$ (PPV = 93\%); and

4. presence of both ermB and mefA genes: non-susceptible with $\mathrm{MIC} \geq 256 \mathrm{mg} / \mathrm{L}$ $(P P V=100 \%)$.

All culture-negative specimens were positive for antibiotic activity (data not shown). Invariably, $>90 \%$ of patients were treated by third-generation cephalosporins. The duration of prior antibiotic therapy ranged from 1-8 days prior to specimen collection, including treatment given before and/or after admission to hospital.

\section{Discussion}

Worldwide, the two most common mechanisms of macrolide resistance in $S$.

pneumoniae are ribosomal methylation encoded by the ermB gene and macrolide efflux encoded by the mefA gene [8]. Both mechanisms are common, but with geographic variations; North American pneumococcal isolates have a greater tendency to harbour $m e f A$, while European isolates more frequently contain erm $B$ and, furthermore, the mef $A$ gene results in low-level resistance whereas the ermB gene, singly or with mefA, is usually associated with high-level resistance $[5,8]$. Few other mutation types (ribosomal mutations in the 23S rRNA gene or in the ribosomal proteins L4 or L22) are rarely found in this organism [18]. However, similar to other studies, all of the erythromycin-non-susceptible isolates $(n=121)$ tested in the current study had at least one of the MRGs (ermB and/or mefA). On the other hand, none of the susceptible isolates $(n=32)$ were positive for either gene. 
In this study, MRGs were detected among all of the CSF specimens with $100 \%$ sensitivity and specificity compared with the results of the corresponding isolates. In addition, each gene (singly or in combination) was consistently associated with a specific range of MICs in the isolates. These findings enabled us to ascertain the possible MICs of pneumococci in culture-negative (pneumococcal antigen/gene detected by Binax/PCR) specimens based on the gene(s) detected.

The success of this study provides the proof-of-principle that MRGs from pneumococci can be detected in culture-negative body fluids (e.g. CSF and ascitic fluid) even after 8 days of antibiotic therapy. This is similar to our previous finding in detecting the orf51 sequence of the antibiotic resistance element ICEHin1056 of Haemophilus influenzae [19]. The study also reveals that PCR-based detection of MRGs can facilitate the tagging of specific pneumococcal specimens (though non-culturable) with an MIC with high precision (PPV > 93\%) for samples containing ermB, mefA or both genes, in addition to classifying them as susceptible and non-susceptible.

The study has limitations, which includes lack of any pneumococcal pleural fluid to test by this method, where macrolide therapy would be of direct relevance. However, our success with CSF and ascitic fluid indicates that PCR will likely work for pleural fluid specimens. Another limitation is that cases with a low pneumococcal DNA load (as revealed by high CT value $>31$ in qPCR) were not been tested by triplex PCR for detection of MRGs. Furthermore, we could not evaluate the clinical impact of early 
detection of MRGs as all of the patients were treated with ceftriaxone from Day 1 of admission.

Use of these methods in addition to conventional culture and antibiotic resistance testing will enable comprehensive surveillance of the prevalence of macrolide resistance among pneumococci, including monitoring of trends in resistance in the context of antibiotic use and/or the impact of PCVs. In populations with high rates of prior antibiotic use, the PCR-based method described here will provide additional information about the epidemiology of MRGs among pneumococci in the specific population, which will guide the empirical and, in most cases, definitive therapy against pneumococcal disease [20]. We are now applying this method to all prospective cases to monitor the comprehensive epidemiology and trends of macrolide resistance in Bangladesh during the post-PCV period.

Acknowledgments: The authors sincerely acknowledge the technical assistance provided by Mr Md. Hafizur Rahman and Mr Md. Shariful Islam.

Funding: This study was partially supported by the World Health Organization [grant no. 201233523], the Bill \& Melinda Gates Foundation [grant no. OPP1096930] and the Child Health Research Foundation. 
Ethical approval: Strains and specimens used in this study were obtained from patients as part of routine care; written informed consent was only obtained from parents or caregivers of all participants for other aspects of the study, including collection of data and the use of specimens for additional laboratory analysis. This study was approved by the Ethical Review Committee of the Bangladesh Institute of Child Health (Dhaka Shishu Hospital, Dhaka, Bangladesh). 


\section{References}

[1] World Health Organization. Estimated Hib and pneumococcal deaths for children under 5 years of age. Geneva, Switzerland: WHO; 2008. http://www.who.int/immunization/monitoring_surveillance/burden/estimates/en/ [accessed 19 January 2017].

[2] Conklin L, Loo JD, Kirk J, Fleming-Dutra KE, Knoll MD, Park DE, et al. Systematic review of the effect of pneumococcal conjugate vaccine dosing schedules on vaccine-type invasive pneumococcal disease among young children. Pediatr Infect Dis J 2014;33(Suppl 2):S109-18.

[3] Saha SK, Hossain B, Islam M, Hasanuzzaman M, Saha S, Hasan M, et al. Epidemiology of invasive pneumococcal disease in Bangladeshi children before introduction of pneumococcal conjugate vaccine. Pediatr Infect Dis J 2016;35:655-61.

[4] Saha SK, Baqui AH, Darmstadt GL, Ruhulamin M, Hanif M, El Arifeen S, et al. Comparison of antibiotic resistance and serotype composition of carriage and invasive pneumococci among Bangladeshi children: implications for treatment policy and vaccine formulation. J Clin Microbiol 2003;41:5582-7.

[5] Song J-H, Dagan R, Klugman KP, Fritzell B. The relationship between pneumococcal serotypes and antibiotic resistance. Vaccine 2012;30:2728-37.

[6] Lynch J 3rd, Zhanel GG. Streptococcus pneumoniae: does antimicrobial resistance matter? Semin Respir Crit Care Med 2009;30:210-38.

[7] Hasanuzzaman M, Islam M, Malaker R, Baqui AH, Darmstadt GL, Whitney CG, et al. Recent emergence of highly macrolide-resistant pneumococcus in 
Bangladesh—relation to antibiotic use and genetic determinants. In: 9th World Congress of the World Society for Pediatric Infectious Diseases; 18-21 November 2015; Rio de Janeiro, Brazil. p. 61.

[8] Song J-H, Chang H-H, Suh JY, Ko KS, Jung S-I, Oh WS, et al. Macrolide resistance and genotypic characterization of Streptococcus pneumoniae in Asian countries: a study of the Asian Network for Surveillance of Resistant Pathogens (ANSORP). J Antimicrob Chemother 2004;53:457-63.

[9] Sutcliffe J, Grebe T, Tait-Kamradt A, Wondrack L. Detection of erythromycinresistant determinants by PCR. Antimicrob Agents Chemother 1996;40:2562-6.

[10] Arifeen SE, Saha SK, Rahman S, Rahman KM, Rahman SM, Bari S, et al. Invasive pneumococcal disease among children in rural Bangladesh: results from a population-based surveillance. Clin Infect Dis 2009;48(Suppl 2):S103-13.

[11] Brooks WA, Breiman RF, Goswami D, Hossain A, Alam K, Saha SK, et al. Invasive pneumococcal disease burden and implications for vaccine policy in urban Bangladesh. Am J Trop Med Hyg 2007;77:795-801.

[12] von Gottberg A, Cohen C, de Gouveia L, Meiring S, Quan V, Whitelaw A, et al. Epidemiology of invasive pneumococcal disease in the pre-conjugate vaccine era: South Africa, 2003-2008. Vaccine 2013;31:4200-8.

[13] Clinical and Laboratory Standards Institute. Performance standards for antimicrobial susceptibility testing; twenty-fourth informational supplement. Document M100-S24. Wayne, PA: CLSI; 2014. 
Pai R, Gertz RE, Beall B. Sequential multiplex PCR approach for determining capsular serotypes of Streptococcus pneumoniae isolates. J Clin Microbiol 2006;44:124-31.

[15] Wang X, Theodore MJ, Mair R, Trujillo-Lopez E, du Plessis M, Wolter N, et al. Clinical validation of multiplex real-time PCR assays for detection of bacterial meningitis pathogens. J Clin Microbiol 2012;50:702-8.

[16] Saha SK, Darmstadt GL, Yamanaka N, Billal DS, Nasreen T, Islam M, et al. Rapid diagnosis of pneumococcal meningitis: implications for treatment and measuring disease burden. Pediatr Infect Dis J 2005;24:1093-8.

[17] Saha SK, Darmstadt GL, Baqui AH, Hossain B, Islam M, Foster D, et al. Identification of serotype in culture negative pneumococcal meningitis using sequential multiplex PCR: implication for surveillance and vaccine design. PLoS One 2008;3:e3576.

[18] Tait-Kamradt A, Davies T, Cronan M, Jacobs M, Appelbaum P, Sutcliffe J. Mutations in 23S rRNA and ribosomal protein L4 account for resistance in pneumococcal strains selected in vitro by macrolide passage. Antimicrob Agents Chemother 2000;44:2118-25.

[19] Saha SK, Darmstadt GL, Baqui AH, Islam N, Qazi S, Islam M, et al. Direct detection of the multidrug resistance genome of Haemophilus influenzae in cerebrospinal fluid of children: implications for treatment of meningitis. Pediatr Infect Dis J 2008;27:49-53.

[20] Nuermberger E, Bishai WR. The clinical significance of macrolide-resistant Streptococcus pneumoniae: it's all relative. Clin Infect Dis 2004;38:99-103. 

Fig. 1. (a) Association of the presence of macrolide resistance genes with minimum inhibitory concentrations (MICs) of erythromycin among 153 Streptococcus pneumoniae isolates from children in Bangladesh. (b) PCR results of corresponding cerebrospinal fluid (CSF) samples of culture-proven cases.

Fig. 2. Distribution of macrolide resistance genes among culture-negative pneumococcal cases. 


\section{7id Figure ia}

50

$$
52 \quad \text { Tsolates N= } 153
$$

3 Isolateg of comasponding CSF, 4. $\mathrm{N}=10$

$2 \prod$ Figure 18

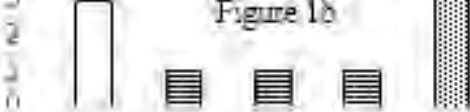
wis te $=256$

$\frac{9}{2} 30$

20

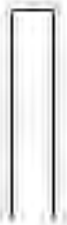

ox.5
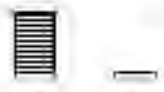

3

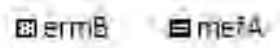

- emmitmét's

arione

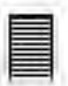

4

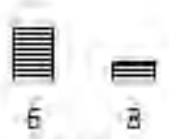

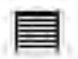

12

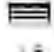

16

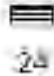

द:स:\%:

E4
(T)

36
हि:क्:?

192

\section{MIC valie of Erythromycii A}

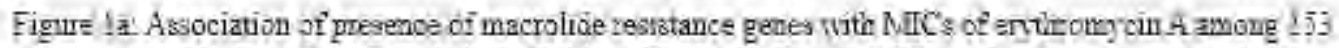
pueumococeal isoiates from Bangiadeshi shildren. Figure Io (Inset) PCR results of ccrrespondug CSF samples of sulture-proven cases: 


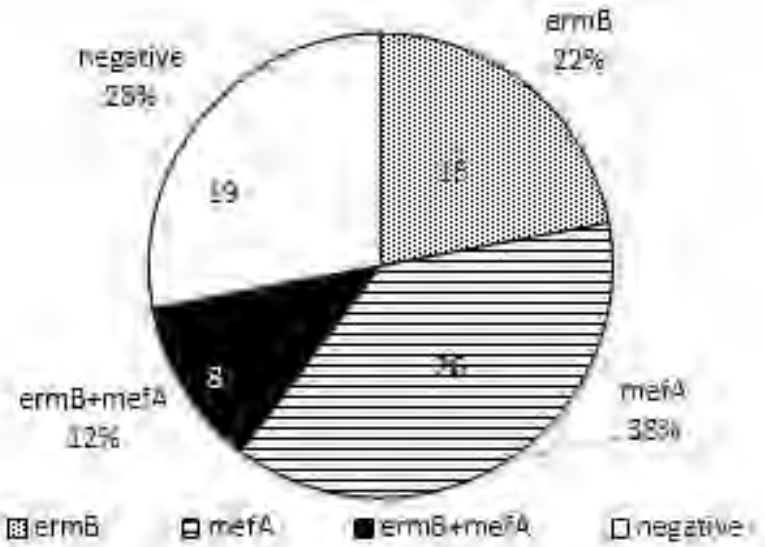

Fugure 2: Diatrioutiou of microlide restance gemes among culture negative pneumorocosl cases. 


\section{Table 1}

Correlation between macrolide resistance genes and erythromycin minimum inhibitory concentrations (MICs) in pneumococcal cases $(n=153)$

\begin{tabular}{|c|c|c|c|c|c|c|c|c|}
\hline MIC (mg/L & $\begin{array}{l}\text { ermB }(n= \\
70)\end{array}$ & $\begin{array}{l}\text { Cum \% of } \\
\text { ermB }\end{array}$ & $\begin{array}{l}\text { mefA }(n= \\
44)\end{array}$ & $\begin{array}{l}\text { Cum \% of } \\
\text { mefA }\end{array}$ & $\begin{array}{l}\text { ermB+mefA }(n= \\
7)\end{array}$ & $\begin{array}{l}\text { Cum \% of } \\
\text { both }\end{array}$ & $\begin{array}{l}\text { None }(n= \\
\text { 32) }\end{array}$ & Total \\
\hline$<0.5$ & 0 & 0 & 0 & 0 & & & 32 & 32 \\
\hline 2 & 0 & 0 & 10 & 23 & & & & 10 \\
\hline 3 & 0 & 0 & 1 & 25 & & & & 1 \\
\hline 4 & 0 & 0 & 10 & 48 & & & & 10 \\
\hline 6 & 0 & 0 & 8 & 66 & & & & 8 \\
\hline 8 & 0 & 0 & 3 & 73 & & & & 3 \\
\hline 12 & 0 & 0 & 6 & 86 & & & & 6 \\
\hline 16 & 0 & 0 & 3 & 93 & & & & 3 \\
\hline 24 & 0 & 0 & 3 & 100 & & & & 3 \\
\hline 64 & 2 & 3 & & & & & & 2 \\
\hline 96 & 1 & 4 & & & & & & 1 \\
\hline 192 & 2 & 7 & & & & & & 2 \\
\hline$\geq 256$ & 65 & 100 & & & 7 & 100 & & 72 \\
\hline
\end{tabular}

